\title{
Effect Of Intermittent And Continuous Training On Body Composition Cardiorespiratory Fitness And Lipid Profile In Obese Adolescents
}

\author{
${ }^{1,}$ Abdessalem Koubaa , ${ }^{2,}$ Hajer Trabelsi , ${ }^{3,}$ Liwa Masmoudi , ${ }^{4,}$ Mohamed \\ Elloumi, ${ }^{5}$ Zouheir Sahnoun, ${ }^{6}$ Khaled M. Zeghal , ${ }^{7}$ Ahmed Hakim \\ 1,2,3,4,5,6,7 Laboratory of Pharmacology, Faculty of Medicine of Sfax, University of Sfax, Tunisia.
}

\begin{abstract}
We assessed the cardiorespiratory responses and exercise tolerance during 12 week training (intermittent vs. continuous) in obese children. Twenty nine obese adolescent were randomly allocated into either the IT or CT group. The CT group performed a 30 to 40 minute continuous exercises a $60 \%$ to $70 \%$ of $v V O 2 m a x$. IT group was instructed to run for 2 min interspersed with recovery periods of one minute. The exercise intensity was $80 \%$ of the vVO2max increased by 5\% every four weeks. Along the training period, the increase of the load was carried out by increasing the intensity and the number of repetitions. At baseline and after 12 weeks of intervention, cardiorespiratory fitness, body composition and lipid parameters were assessed. Improvement of Weight and Fat mass were significantly lower during the intermittent exercise (Weight (kg) $=81.1 \pm 13.8 \mathrm{vs} .79 .1 \pm 13.3$ and Fat mass $=33.8 \pm 6.7 \mathrm{vs} .31 .8 \pm 7.1$ respectively) compared to the continuous exercise in the obese adolescent (Weight $=80.7 \pm 13.2$ vs. $75.8 \pm 11.2$ and fat mass $=33.7 \pm 6.7$ vs. $28.8 \pm 5.1$ respectively). After the continuous training program, significant beneficial effects on the $H D L-C_{(m m o l . l-1)}(0.97$ \pm 0.06 vs. $1.12 \pm 0.1)$, ratio $L D L-C / H D L-C(2.93 \pm 0.22$ vs. $2.39 \pm 0.13)$, ratio TC/HDL-C $(4.57 \pm 0.19$ vs. $3.92 \pm 0.20)$. Improved capacity cardiorespiratory best of IT versus CT, at the Resting. HR (beats. $\left.\mathrm{m}^{-1}\right)(83 \pm 4$ vs $78 \pm 3$ and

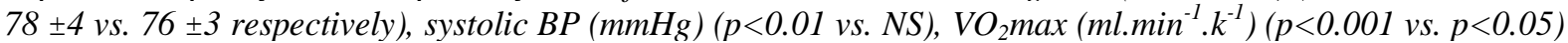
and recovery index $(p<0.01$ vs. $p<0.05)$. Greater improvement in cardiorespiratory fitness of intermittent group compared to the continuous group. The other subjects who completed the continuous training program have improved their lipid profiles and their body composition more that the group intermittent.
\end{abstract}

Keywords: intermittent training; continuous training; body composition; cardiorespiratory fitness; lipid profiles; obese adolescent.

\section{Introduction}

Obesity during childhood and adolescence has been identified as a major health problem throughout the world [1]. It has been associated with a reduced exercise tolerance and a decrease of cardiopulmonary function as well as an alteration of peripheral muscles responses [2-3]. Decreased physical activities and sedentary life style were the most important factors contributing in the development of paediatric obesity [4]. Consequently, exercise training was often included in weight-loss programs with energy restriction. It was shown previously, that the practice of a regular exercise by obese subjects (with different obesity classes) improves their body composition and their functional capacities [5-6]. The benefits of regular exercise are well documented [7], but the rationale underlying the exercise mode recommendations for specific health benefits remains unclear due in large part to the sparse scientific data supporting these recommendations. We need require clear exercise guidelines based upon sufficient evidence from which to prescribe the most effective exercise plan [8]. Most studies consider that continuous training to be beneficial for maintaining cardiovascular fitness. However, exercising with intervals may be more effective [9]. In addition, other researchers have suggested that intermittent exercise of moderate intensity with at least 10 minutes duration accumulating 30 minutes per day(3 times / session / 30 min per day), provided cardiovascular benefits [9]. DeBusk et al., [10], observed that, subjects who exercised in intermittent sessions experienced gain in physical fitness and improvement in blood lipid profile. Previous studies showed that the intermittent bouts of walking provided improvement in aerobic fitness, body composition, plasma lipoprotein and blood pressure compared to continuous or long session of physical training [11]. Short bouts of intermittent exercise training lead to a significant improvement in cardiovascular fitness as compared to the longer continuous bout of similar duration [12]. On the other hand, a continuous training program for $70-80 \%$ of maximum heart rate for 30 min / day, 5 days / week showed an increase in aerobic capacity, improved blood lipid profiles and improved aspects of physiological well-being, sedentary middle- aged adults [13]. Regular physical activity reduces, also, the risk of cardiovascular disease [11]. therefore the present study was undertaken with a view to investigate the 
effectiveness of continuous and intermittent exercise training on body composition, the lipid profile and the cardiorespiratory fitness of obese adolescent boys. We hypothesize that the continuous training would further improve body composition, and lipid profile while training intermittent yield results more relevant cardiorespiratory capacity

\subsection{Subjects}

\section{Methods}

Twenty nine for obese adolescents (boys), in good health participated in this study. Their mean values of age, height and weight were respectively $13 \pm 08$ years, $1.62 \pm 0.08 \mathrm{~m}, 81.2 \pm 13.7 \mathrm{~kg}$. After receiving a complete verbal description of protocol, risks and benefits of the study, the subjects provided written consent to an experimental protocol approved by the Researsh Ethics Committee of the Faculty Medicine's, from University of Sfax in Tunisia. A stripling was defined as obese when his Body Mass Index (BMI) is superior to the 97th percentile defined by Rolland- Cachera et al, [14]. A draw has divided the subjects into two groups. The first group of 15 adolescents (CT) participates in continuous training program. The second group of 14 adolescents (IT) realizes the program of intermittent training.

\subsection{Materials and measured parameters \\ 2.3. Anthropometric measures}

Mass $(\mathrm{kg})$ of the subjects was measured with a calibrated electronic scale and standing height $(\mathrm{m})$ was measured with a stadiometer fixed. Similarly, waist circumference $(\mathrm{cm})$ was also measured using a tape. BMI was calculated for each subject using the following formula: weight / $\operatorname{Size}^{2}\left(\mathrm{~kg} \cdot \mathrm{m}^{-2}\right)$.Body fat $(\%)$ was estimated from four skinfolds (supra-iliac, biceps, triceps and subscapularis) according to Durnin and Womersley formula [15]. The measurement of these skinfolds was conducted by a clamp Harpenden. This fat is used to calculate the Lean Mass (LM) by the formula of Hallynck et al, [16]. $\mathrm{MM}=(1,10 *$ weight $(\mathrm{kg}))-128 *\left[\right.$ weight $\left.^{2} /(100 * \text { height }(\mathrm{m}))^{2}\right]$

\subsection{A stress test}

Measures of $\mathrm{VO}_{2}$ max and the recovery ability post-exercise will be discussed at the end of the test with Lurking triangular wheels (COSMED Pulmonan-Function Equipment 37 Via dei Piani di monte Savello I00040 Rome ITALY).This dynamic test, until the maximum fatigue, consists in increasing the speed of $1 \mathrm{~km} /$ time every $2 \mathrm{~min}$, after warm up for $5 \mathrm{~min}$ with a speed of $6 \mathrm{~km} /$ time. Heart rate and $\mathrm{VO}_{2}$ during the test and recovery will be measured using an analyzer (version 1.2 PRO Fit mate COSMED)

\subsection{Determination of serum lipids}

Venous blood samples (ante-cubital vein) were performed in dry tubes under basal conditions ( $8 \mathrm{am}$ morning). After centrifugation, the sera were frozen at $-80^{\circ} \mathrm{C}$ until analysis. Total cholesterol (TC), triglycerides (TG), high-density lipoprotein cholesterol (HDL-C), and glucose levels were measured in all subjects before and after programs after 12 hours fasting using standardized techniques described by Wegge JK [17]. Low-density lipoprotein cholesterol (LDL-C) was calculated as described by the Friedewald formula [18].

\subsection{Exercise Training Protocols.}

The subjects were divided into two groups and subjected to two training programs (interval training (IT), continuous training (CT)). The training lasted 12 weeks with three weekly sessions. The intensity was monitored as a function of time and the distance traveled

2.7 Intermittent training: Intermittent group was instructed to run for $2 \mathrm{~min}$ interspersed with recovery periods of one minute. The exercise intensity was $80 \%$ of the $\mathrm{vVO}_{2} \max$ increased by $5 \%$ every four weeks. Along the training period, the increase of the load was carried out by increasing the intensity and the number of repetitions

2.8 Continuous training: Training was carried out continuously at $60 \%$ of $\mathrm{vVO}_{2} \max$ (first 4 weeks), $65 \%$ of $\mathrm{vVO} 2 \mathrm{max}$ (second 4 weeks) and $70 \%$ of $\mathrm{vVO}_{2} \max$ ( $3 \mathrm{rd} 4$ weeks).Progress was controlled by increasing the intensity and duration of exercise in order to have the same training load between groups trained.

\subsection{Statistical analysis}

All data were presented as means values and standard deviation.

A paired Student's t-test was used to analyze within group changes. The differences between the two groups (IT) and (CT) were compared by unpaired Student's t-tests. Tukey's post hoc test was used to compare means and to evaluate the relationships between various parameters. Spearman's correlation analysis was performed. Analysis was performed using StatView software and the significance threshold was set at $p<0.05$. 


\section{Results \\ 3.1. Two protocols effects on the anthropometric characteristics \\ Basic anthropometric characteristics of our population and their changes during the training period are reported in Table 1. Groups (IT) and (CT) were similar in age, size and exhibited similar adiposity. This was evaluated by BMI, the fat and waist circumference (WC).The training period of 3 months induces a modification of all parameters of body composition of our subjects. However, this varies depending on group.}

\subsection{Intra-group body weight changes}

Table 1 shows a significant decrease in body weight of subjects in our population. The subjects (CT) weighing $80.7 \pm 13.2 \mathrm{~kg}$ before and $75.8 \pm 11.2 \mathrm{~kg}$ after the training period, ( a decrease of $-4.9 \pm 2.1 \mathrm{~kg}, \mathrm{p}$ $<0.01$ ). For group (IT), this decrease is less important. Indeed, body weight adolescents pass of $81,1 \pm 13.8 \mathrm{~kg}$ before to $79.1 \pm 13.3 \mathrm{~kg}$ after this period ( a decrease of $-2.0 \pm 0.5 \mathrm{~kg}, \mathrm{p}<0.05$ ).

\subsection{Intra-group BMI changes in}

Table 1 shows a significant reduction in BMI in our subjects during the training period. In the group (CT), this index pass of $30.8 \pm 2.9 \mathrm{Kg} \cdot \mathrm{m}^{-2}$ before, to $28.5 \pm 2.6 \mathrm{Kg} \cdot \mathrm{m}^{-2}$ after the training program, or a loss of $-2.3 \mathrm{Kg} \cdot \mathrm{m}^{-2} \pm 0.6$ ( $\left.\mathrm{p}<0.01\right)$. In the group (IT), a decrease of BMI is less marked, but it is significant. Thus, the values of this index pass of $30.2 \pm 3.6 \mathrm{Kg} . \mathrm{m}-2$ before to $29.4 \pm 3.1 \mathrm{Kg} . \mathrm{m}^{-2}$ after the training program, a decrease of $-1.2 \pm 0.4 \mathrm{Kg} \cdot \mathrm{m}^{-2}(\mathrm{p}<0.05)$.

\subsection{Intra-group fat mass changes}

The training program induces also a decrease in body fat in all our subjects. It pass in the group (CT) of $33.7 \pm 6.7 \mathrm{~kg}$ before to $28.8 \pm 5.1 \mathrm{~kg}$ after the training program, a decrease of $-4.9 \pm 2.4 \mathrm{~kg}(\mathrm{p}<0.01)$. The same is observed for the group (IT), in which values of fat are $31.8 \pm 7.1 \mathrm{~kg}$ after against $33.8 \pm 6.7 \mathrm{~kg}$ before the training period, which corresponds to a decrease of $-2.0 \pm 0.5 \mathrm{~kg}(\mathrm{p}<0.05)$.

\subsection{Intra-group fat free mass changes}

The training period does not alter lean mass in all our subjects. However, a slight increase but not significant of this mass was observed in group (CT) $(\mathrm{p}=0.062)$. The values pass of $46.4 \pm 7.8 \mathrm{~kg}$ before to 47.1 $\pm 7.3 \mathrm{~kg}$ after this period.

\subsection{Intra-group waist circumference changes}

Our program induces, also, a decrease (WC) of all our subjects. This varies depending on group. It pass in the group (CT) of $100.1 \pm 12.3 \mathrm{~cm}$ before to $94.3 \pm 8.7 \mathrm{~cm}$ after continuous training, a decrease of $-5.8 \pm$ $3.7 \mathrm{~cm}(\mathrm{p}<0.01$ ). The same is observed for group (IT), the decrease of waist circumference is less marked. its values pass of $98.2 \pm 9.4 \mathrm{~cm}$ before to $96.3 \pm 9.9 \mathrm{~cm}$ after training intermittent, or a loss of $-1.9 \pm 5.4 \mathrm{~cm}(\mathrm{p}=$ $0.06)$.

\subsection{Two protocols effects on the lipid profile}

Lipid parameters values in pre-and post-program are summarized in Table 2. Before our program, lipid basal plasma was significantly similar in all of our subjects. Our continuous training program induced a significant decrease in plasma concentrations of TG, LDL-C and TC. Similarly, we recorded in these same subjects $(\mathrm{CT})$, lowered reports LDL-C/HDL-C and CT/ HDL-C $(\mathrm{p}<0.01)$. The decrease in these parameters were less marked in group (IT) ( $\mathrm{p}<0.05$ ). However, in this group no significant change was recorded at TC and LDL-C. The HDL-C/TG ratio were significantly increase after our continuous training $(0.71 \pm 0.11$ before versus $0.86 \pm 0.09$ after $(\mathrm{p}<0.01))$. Finally, intermittent and continuous training showed a significant increase in HDL-C ( $<<0.05$ and $\mathrm{p}<0.01$ respectively).

\subsection{Two protocols effects on the cardiorespiratory fitness}

Cardio-respiratory parameters Changes in both groups of our population before and after training are summarized in Table 3. For groups (CT) and (IT) a resting heart rate decrease is significant ( $p<0.05$ and $p$ $<0.01$ respectively).A systolic blood pressure (SBP) decrease follows the same profile. This is more marked in group (IT) compared with those in group (CT). It is respectively $131 \pm 2$ after versus $134 \pm 3$ before and $131 \pm 3$ before versus $129 \pm 3$ after).

Similarly, we recorded in these groups a diastolic blood pressure (DBP) lowered, but is significant only for subjects who completed the continuous training program (IT) $(\mathrm{p}<0.05)$. Statistical analysis revealed a significant improvement in VO2max, maximal aerobic speed (MAS) and recovery index (RI) in both groups: (CT) (p $<0.05, \mathrm{p}<0.01$ and $\mathrm{p}<0.05$ respectively) (IT) $(\mathrm{p}<0.001, \mathrm{p}<0.001$ and $\mathrm{p}<0.01$ respectively). 


\section{Discussion}

At the end of the training period, changes in body composition were illustrated. These are characterized by a reduction in body weight, BMI and body fat while maintaining Fat free mass. Also, the body mass loss recorded in our study varies depending the groups. It is higher in group (CT) compared with those in group (IT). The group (CT) has lost $-4.9 \pm 2.1 \mathrm{~kg}$, while the group (IT) has lost only $-2.0 \pm 0.5 \mathrm{~kg}$. The first observation is that, as in adults, studies on the physical exercise effect on the weight of the adolescent show sometimes contradictory results [19-20]. Our results about weight loss group (IT) are similar to those published by Arnt Erik Tjonna and al [21] and differ from those reported by Coquart JB and al [22]. It seems weight loss is modest although significant in this type of study. Despite the discrepancies between the results; it seems that the continuous exercise provides a significant improvement in weight loss [22-23]. This observation is verified in group subjects (CT) showed an important decrease in body mass with a loss about $-4.9 \pm 2.1 \mathrm{~kg}$. Our observations join, therefore, the postulate of Arnt Erik Tjonna and al [21], who observed in adults under 16 weeks of continuous exercise a significant decrease of $-3.6 \mathrm{~kg}$ bodyweight. The magnitude of the weight loss is consistent with other investigations that used exercise and represents a $2.1 \%$ decrease for continuous program and $1.0 \%$ decrease for intermittent program [24-25]. Our program also causes a decrease in BMI and body fat in all our subjects. Thus, their means BMI pass of $30.5 \mathrm{~kg} \cdot \mathrm{m}^{-2}$ before program to $29 \mathrm{~kg} . \mathrm{m}^{-2}$ after this period, which corresponds to an average decrease of $-1.5 \mathrm{~kg} . \mathrm{m}^{-2}$. This BMI decrease was important in group subjects (CT) that in group (IT) and different from that observed by Coquart JB and al [22] and similar than reported by Lazzer and al [6-26]. Similarly, the fat mass in the two combined groups passes of $33.8 \mathrm{Kg}$ before to $30.3 \mathrm{~kg}$ after the program, or $-3.5 \mathrm{~kg}$ loss. Similar findings were reported by Lazzer and al [26], on a period of 9 months. Although comparisons of our results with other are difficult because of the difference of measurement methods, it appears that our training program allows significant improvements in body composition that are important than previous studies. Separating the two groups, we find that the fat mass loss of the group (CT) was significantly higher than that observed in group (IT). It is of $-4.9 \pm 2.4 \mathrm{~kg}$ in $(\mathrm{CT})$ then it is only $-2.0 \pm 1.6 \mathrm{~kg}$ in (IT) $(\mathrm{p}<0.01$ and $\mathrm{p}<0.05$ respectively) .

Also, our program has induced changes in waist circumference of all our subjects. Its mean value, all combined groups passes of $99.2 \mathrm{~cm}$ before to $95.3 \mathrm{~cm}$ after the program, a decrease of $-3.9 \mathrm{~cm}$. Separating the two groups we found that the waist circumference loss is significant only in subjects of (CT) compared to that of (IT). This loss is of the order of $-5.8 \pm 3.8 \mathrm{~cm}$ in (CT) but she is only that of $-1.9 \pm 4.6 \mathrm{~cm}$ in (IT). Our results are consistent therefore with the advanced of Arnt Erik Tjonna and al [21] after 16 weeks intervention. Finally, our training program produces no change in fat free mass of all our subjects, except group (CT) with a nonsignificant increase $(\mathrm{p}=0.58)$. In addition, our study examining the effects of two protocols, namely the continuous and intermittent training, not only on the body composition and lipid profile but also on the cardiorespiratory fitness for obese adolescents Tunisians. In our study the interval training improves one hand, the ability to oxidize lipids at exercise and secondly, it induces a decrease in triglycerides and increased the HDL cholesterol. Our results are similar those reported by JE Donnelly and al [23], showed that a change in HDL-C following a continuous or intermittent training However, in group subjects (CT), we found a greater increase of HDL-C values and of ratio of HDL cholesterol to triglycerides. According to our study, the significant reduction in plasma concentrations of the ratio TC / HDL-C, and LDL-C/HDL-C is mainly due to the choice of continuous training. Intermittent training program did not induce significant changes in the values of total cholesterol (TC) and LDL cholesterol (LDL-C), but produced an increase in HDL cholesterol (HDL-C) values and a decrease in the ratio of TC / HDL-C. Our results are consistent with the postulate of Arnt Erik Tjonna and al [21], which showed no change in the values of TG, LDL-C and TC after an intermittent and continuous training. It appears that continuous training, although it did not significantly affect the values of TC and LDL-C, it induces significant changes of ratio TC / HDL-C. Our study provides a further demonstration on exercises choice that could be prescribed and recommended in obese subjects. The continuous training improves the body composition, lipid profile. This type of training seems to have a more sustained than the intermittent The result revealed that both intermittent and continuous groups had a significant decline in blood pressure and heart rate of both continuous and intermittent training, reduction was greater in intermittent group (IT) than in continuous group. However, both types of training induced a decrease in resting heart rate, systolic blood pressure (SBP) and diastolic blood pressure (DBP) and an increase in $\mathrm{VO}_{2}$ max and recovery index. Yet, in group (IT), we found a greater improvement of all recorded values. Our results are similar to those of [10-27], which showed almost the same change. In our study, the relative $\mathrm{VO}_{2}$ max of both the continuous and intermittent training groups improved significantly, Continuous and intermittent training improved the $\mathrm{VO}_{2}$ max of sedentary persons was observed in previous studies [28-29]. Cardio respiratory endurance is defined as ability to sustain prolonged, rhythmic exercise. It is related with the highest rate of oxygen consumption obtainable by the active muscle during maximal or exhaustive exercise [30]. Both the exercise training program increased the $\mathrm{VO}_{2}$ max reflected an increase in cardio-respiratory system efficiency. Previous researchers also 
observed that both the continuous and intermittent exercise training program were able to improve the $\mathrm{VO}_{2}$ max [10-28-32]. But in our study, we observed that the degree of improvement in $\mathrm{VO}_{2}$ max was higher after intermittent training as compared to the continuous.

\section{Conclusion}

That exercise training intermittent or continuous, in the presence of changes in body composition, improves the lipid profile and cardiorespiratory fitness. It appears from this study that 12 weeks of intermittent or continuous training has a positive additional effect on the body of our subjects. These results in a greater improvement in cardiorespiratory fitness of intermittent group compared to the continuous group. The other subjects who completed the continuous training program have improved their lipid profiles and their body composition more that the group intermittent. These findings lead us to consider a combined training start first with training intermittent aimed to improve cardiorespiratory fitness to ensure a better fit and then enchained with continuous training to foster better improvement to adolescent health in overweight or obese.

\section{Bibliography}

[1] Speiser PW, Rudolf MCJ, Anhalt H, On behalf Obesity Consensus Working Group. Consensus statement: Childhood obesity.Journal of Clinical Endocrinology and Metabolism 2005; 90: 1871-1887.

[2] Kimm SY, Glynn NW, Obarzanek E, Kriska AM, Daniels SR, Barton BA, Liu K. Relation between the changes in physical activity and body-mass index during adolescence: A multicentre longitudinal study. Lancet 2005; 366 (9482): 301-307.

[3] Norman AC, Drinkard B, McDuffi e JR, Ghorbani S, Yanoff LB, Yanovski JA. Influence of excess adiposity on exercise fitness and performance in overweight children and adolescents. Pediatrics 2005; 115: e690-e696.

[4] Reybrouck T, Mertens L, Schepers D, Vinckx J, Gewillig M. Assessment of cardiorespiratory exercise functions in obese children and adolescents by body massindependent parameters. European Journal of Applied Physiology and Occupational Physiology 1997; 75 (6): $478-483$.

[5] Brandou F, Dumortier M, Garandeau P, Mercier J, Brun JF. Effects of two-month rehabilitation program on substrate utilization during exercise in obese adolescents. Diabetes Metab 2003; 29: 20-27.

[6] Lazzer S, Boirie Y, Poissonnier C, Petit I, Duche P, Taillardat M, Meyer M, Vermorel M. Longitudinal changes in acti vity patterns, physical capacities, energy expenditure, and body composition in severely obese adolescents during a multidisciplinary weightreduction program. International Journal of Obesity (London) 2005; 29: 37-46.

[7] Andersen RE, and Jakicic JM. Interpreting the physical activity guidelines for health and weight management. Journal of physical activity \& health, 2009 6: 651-656.

[8] Ogden CL, Carroll MD, Curtin LR, McDowell MA, Tabak CJ, and Flegal KM. Prevalence of overweight and obesity in the United States, 2006. JAMA 295: 1549-1555.

[9] Lyndon JO, Jacob B, Leslie I, Katzel and Andrew PG (2005). Exercice training and cardiovascular health with aging. Clinical Geriatrics 13(4) 40-46

[10] DeBusk RF, Stenestrand U, Sheehan M and Haskell WL (1990). Training effects of long versus short bouts of exercise in healthy subjects. Amercan Journal of Cardiology 65(15) 101-03.

[11] Haskell WL, Lee IM, Pate RR, Powell KE, Blair SN, Franklin BA, Macera CA, Heath GW, Thompson PD and Bauman A (2007). Physical activity and public health: updated recommendation for adults from the American College of Sports Medicine and the American Heart Association. Medicine and Science in Sports and Exercise 39(8) 1423-34.

[12] Macfarlane DJ, Taylor LH and Cuddihy TF (2006). Very short intermittent vs continuous bouts of activity in sedentary adults. Preventive Medicine 43(4) 332-336.

[13] Murphy M, Nevill A, Neville C, Biddle S and Hardman A (2002). Accumulating brisk walking for fitness, cardiovascular risk, and psychological health. Medicine and Science in Sports and Exercise 34(9) 1468-1474.

[14] Rolland-Cachera MF, Cole TJ, Sempé M, Tichet J, Rossignol C, Charraud, A. Body mass index variations: centiles from birth to 87 yearth. Eur J Clin Nutr 1991; 45: 13-21.

[15] Durnin JVGA, Womersley J. Body fat assessed from total body density and its estimation from skinfold thickness: measurements on 481 men and women aged from 16 to 72 years. Br J Nutrition. 1974; 32: 77-97.

[16] Hallynck TH; Soep HH et al. should clearance be normalised to body surface or to lean body mass? Br J Clin Pharmacol.1981; 11; 523-526.

[17] Wegge JK, Roberts CK, Ngo TH, et al. Effect of diet and exercise intervention on inflammatory and adhesion molecules in postmenopausal women on hormone replacement therapy and at risk for coronary artery disease. Metabolism 2004; 53:77-81.

[18] Friedewald WT, Levy RI, Fredrickson DS. Estimation of the concentration of low-density lipoprotein cholesterol in plasma, without use of the preparative ultracentrifuge. Clin Chem 1972; 18:499-502.

[19] Sasaki J, Shindo M, Tanaka H, Ando M, Arakawa K. A long-term aerobic exercise program decreases the obesity index and increases the high density lipoprotein cholesterol concentration in obese children. Int J Obes 1987, 11: 339-345.

[20] Epstein LH, Valoski AM, Vara LS, Mccurley J, Wisniewski L. Effects of decreasing sedentary behavior and increasing activity on weight change in obese children. Health Psychol 1995; 14: 109-115.

[21] Arnt Erik Tjonna,; Sang Jun Lee, Tomas O. Stolen, Sonia M. Najjar, Ulrik Wisloff, Aerobic Interval Training Versus Continuous Moderate Exercise as a Treatment for the Metabolic Syndrome American heart association journal July. 2008; 118: $346-354$.

[22] Coquart JB, Lemaire C, Dubart AE, Luttembacher DP, Douillard C, Garcin M. Intermittent versus continuous exercise: effects of perceptually lower exercise in obese women Med Sci Sports Exerc. 2008; 40(8):1546-53.

[23] J E Donnelly1, D J Jacobsen1, K Snyder Heelan1, R Seip2 and S Smith3 The effects of 18 months of intermittent vs continuous exercise on aerobic capacity, body weight and composition, and metabolic fitness in previously sedentary, moderately obese females International Journal of Obesity (2000) 24, 566-572.

[24] Ballor DL, Keesey RE. A meta-analysis of the factors affecting exercise-induced changes in body mass, fat mass, and fat-free mass in males and females. Int J Obes1991; 15: 717:726.

[25] Epstein LH, Wing RR. Exercise and weight. Addictive Behaviors 1980; 5: $371 \square 388$. 
[26] Lazzer S, Boirie Y, Montaurier C, Vernet J, Meyer M, Vermorel M. A weight reduction program preserves fat-free mass but not metabolic rate in obese adolescents. Obes Res 2004; 12: 233-240.

[27] Wahab B Lawal1, Venkatseswarul Kankanala2 : Effets de l'entraînement intermittent et continu de la pression artérielle des adolescents et des enfants nigérians Br J Sports Med 2010.078725.94.

[28] Hardman AE (2001). Issues of fractionization of exercise (short vs long bouts). Medicine and Science in Sports and Exercise 33(6) Suppl, S421-7

[29] Frédéric N. Daussin 1, Joffrey Zoll 1 , Stéphane P. Dufour 1 ,Elodie Ponsot 1, Evely Lonsdorfer Loup 1 , Stéphane Doutreleau 1 , Bertrand Mettauer 1, 2, François Piquard 1, Bernard Geny 1, et Ruddy Richard 1: Effect of interval versus continuous training on cardiorespiratory and mitochondrial functions: relationship to aerobic performance improvements in sedentary subjects; Am J Physiol Regul Integr Comp Physiol; 2008.295:R264-R272.

[30] Wilmore JH, Costill DL and Kenney WL (2008). Physiology of Sport and Exercise, 3rd Ed., Human Kinetics, Leed (UK) 53-59.

[31] Schmidt WD, Biwer CJ and Kalscheuer LK (2001). Effects of long versus short bout exercise on fitness and weight loss in overweight females. Journal of American College of Nutrition 20(5) 494-501.

[32] Jakicic JM, Winters C, Lang W and Wing RR (1999). Effects of intermittent exercise and use of home exercise equipment on adherence, weight loss, and fitness in overweight women: a randomized trial. Journal of American Medical Association 282(16) $1554-60$.

\section{Results}

Table1. Anthropometric characteristics before and after the 3-month program in CT and IT groups (mean \pm standard deviation)

\begin{tabular}{lccccc}
\hline \multirow{2}{*}{ Parameters } & \multicolumn{2}{c}{ CT $(\mathrm{n}=15)$} & & \multicolumn{2}{c}{ IT $(\mathrm{n}=14)$} \\
\cline { 2 - 3 } \cline { 5 - 6 } \cline { 5 - 6 } Age (y) & Before & After & & Before & After \\
Height (m) & $12,9 \pm 0,5$ & & & $13 \pm 0,8$ & \\
Weight (kg) & $1,62 \pm 0,08$ & & & $1,64 \pm 0,05$ & \\
BMI (kg/m-2) & $80,7 \pm 13,2$ & $75,8 \pm 11,2 * *$ & & $81,1 \pm 13,8$ & $79,1 \pm 13,3 *$ \\
Fat mass (kg) & $30,8 \pm 2,9$ & $28,5 \pm 2,6 * *$ & & $30,2 \pm 3,6$ & $29,4 \pm 3,1 *$ \\
Fat free mass (kg) & $33,7 \pm 6,7$ & $28,8 \pm 5,1 * *$ & & $33,8 \pm 6,7$ & $31,8 \pm 7,1 *$ \\
waist circumference (cm) & $46,4 \pm 7,8$ & $47,1 \pm 7,3$ & & $47,3 \pm 7,5$ & $46,8 \pm 5,9$ \\
\hline \hline
\end{tabular}

Legend: CT, continuous training; IT, intermittent training; BMI, body mass index; $* \mathrm{p}<0,05 ; * * \mathrm{p}<0,01 ; * * *$ $\mathrm{p}<0,001$ after versus before program. 
Table2. Blood chemistry after versus before three month program in CT and IT groups (mean \pm standard deviation)

\begin{tabular}{lccccc}
\hline \hline & \multicolumn{2}{c}{ CT $(\mathrm{n}=15)$} & & \multicolumn{2}{c}{ IT $(\mathrm{n}=14)$} \\
\cline { 2 - 3 } \cline { 5 - 6 } Parameters & Before & After & & Before & After \\
\hline Triglycerides (mmol/l) & $1,38 \pm 0,32$ & $1,31 \pm 0,2$ & & $1,35 \pm 0,16$ & $1,27 \pm 0,11^{*}$ \\
Cholesterol (mmol/l) & $4,48 \pm 0,09$ & $4,43 \pm 0,06$ & & $4,37 \pm 0,11$ & $4,32 \pm 0,1$ \\
HDL-C (mmol/l) & $0,97 \pm 0,06$ & $1,12 \pm 0,1 * *$ & & $0,99 \pm 0,04$ & $1,03 \pm 0,08^{*}$ \\
LDL-C (mmol/l) & $2,87 \pm 0,13$ & $2,70 \pm 0,21$ & & $2,75 \pm 0,15$ & $2,7 \pm 0,13$ \\
LDL-C/HDL-C & $2,93 \pm 0,22$ & $2.39 \pm 0,13 * * *$ & & $2,78 \pm 0,23$ & $2,64 \pm 0,27^{*}$ \\
Ratio TC/HDL-C & $4,57 \pm 0,19$ & $3.92 \pm 0,20 * *$ & & $4,41 \pm 0,25$ & $4,21 \pm 0,32^{*}$ \\
Ratio HDL-C/Triglycerides & $0,71 \pm 0,11$ & $0,86 \pm 0,09 *$ & & $0,74 \pm 0,15$ & $0,78 \pm 0,13$ \\
\hline \hline
\end{tabular}

Legend: HDL-C, height density lipoprotein cholesterol; LDL-C, low density lipoprotein cholesterol; TC, Total cholesterol; * $\mathrm{p}<0,05 ; * * \mathrm{p}<0,01 ; * * * \mathrm{p}<0,001$ after versus before program.

Table3. Cardio-pulmonary data of adolescents before and after the 3-month program in CT and IT groups (mean \pm standard deviation)

\begin{tabular}{|c|c|c|c|c|}
\hline \multirow[b]{2}{*}{ Parameters } & \multicolumn{2}{|c|}{$\mathrm{CT}(\mathrm{n}=15)$} & \multicolumn{2}{|c|}{ IT $(n=14)$} \\
\hline & Before & After & Before & After \\
\hline Resting. HR(beats. $\mathrm{m}^{-1}$ ) & $78 \pm 4$ & $76 \pm 3 *$ & $83 \pm 4$ & $78 \pm 3 * *$ \\
\hline Systolic. BP (mmHg) & $131 \pm 3$ & $129 \pm 3 *$ & $134 \pm 3$ & $131 \pm 2 * *$ \\
\hline Diastolic. BP (mmHg) & $85 \pm 6$ & $83 \pm 3$ & $87 \pm 5$ & $84 \pm 3^{*}$ \\
\hline $\operatorname{VO} 2 \max \left(\mathrm{ml} \mathrm{kg}^{-1} \min ^{-1}\right)$ & $37,5 \pm 1,6$ & $39,2 \pm 3,2 *$ & $38,7 \pm 1,2$ & $42,9 \pm 1,7 * * *$ \\
\hline MAS $(\mathrm{km} / \mathrm{h})$ & $10,5 \pm 0,9$ & $11,2 \pm 0,5^{* *}$ & $11,1 \pm 0,3$ & $12,5 \pm 0,4 * * *$ \\
\hline Recovery index (RI) & $15,8 \pm 0,7$ & $17,1 \pm 0,8^{*}$ & $14,8 \pm 1$ & $16,4 \pm 1,8 * *$ \\
\hline
\end{tabular}

Legend: IT, intermittent training; CT, continuous training; Resting. HR, resting hear rat; Systolic. BP, systolic blood pressure; Diastolic. BP, diastolic blood pressure; $\mathrm{VO}_{2} \mathrm{max}$, maximum oxygen uptake; MAS, maximal aerobic speed $* \mathrm{p}<0,05 ; * * \mathrm{p}<0,01 ; * * * \mathrm{p}<0,001$ after versus before program. 